\title{
Experimental Evaluation of Lithium Detection Limits by Windowless SDD EDXS and EELS in a Binary Al-Li Alloy Powder
}

\author{
Vladimir Oleshko ${ }^{1}$, Eli Fastow ${ }^{2}$, Keana Scott $^{1}$ and John Cumings ${ }^{2}$ \\ ${ }^{1}$ NIST, Gaithersburg, Maryland, United States, ${ }^{2}$ University of Maryland, College Park, Maryland, United \\ States
}

Spatially-resolved nanoscale detection and quantification of lithium in emerging materials are critical for such key technology application areas as renewable energy, transportation, aerospace, organic synthesis and pharmacy. $\mathrm{Li}$ is the lightest element that can generate characteristic X-rays by electron interaction, but $>99 \%$ of Li X-ray photons are expected to be absorbed in a $0.3 \mu \mathrm{m}$ thick polymer window $[1,2]$. Windowless silicon drift (SDD) energy-dispersive X-ray (EDX) detectors with low-noise electronics and more efficient geometry designed to collect X-rays under low-kV conditions down to $20 \mathrm{eV}$ have advanced ultra-soft X-ray light element analysis by eliminating the absorption of Li Ka and other soft X-rays. Although lithium detection in Li metal foils by SDD EDXS was demonstrated recently [1, 2], detection challenges remain [2,3]. Li and its compounds are often highly reactive and, upon being exposed to air, readily react with it. The escape depth of $\mathrm{Li}$ is less than $20 \mathrm{~nm}$ and, as the surface accumulates the oxidation products, the Li Ka X-rays are increasingly absorbed in the oxidized layer. Additionally, the transition from the $\mathrm{Li} \mathrm{Ll}$ to $\mathrm{K}$ shells is not allowed by the selection rule. Unless bonding interaction causes the $\mathrm{Ll}$ electron to take on more L2,3 characteristics, Li X-ray generation therefore cannot occur $[1,3]$.

In this work, we report on identification of lithium detection limits evaluated by windowless SDD EDXS and electron energy-loss spectroscopy (EELS) in a commercial Al-Li alloy (Li mass fraction of $20 \%$ ) powder [2]. Prior to EDX analyses with a windowless SDD EDXS detector installed on a dual beam focused ion beam scanning electron microscope (FIB)/SEM), the microstructure and local chemical compositions of the powders were characterized using low-voltage field-emission SEM, conventional and phase-contrast high-resolution transmission EM (CTEM/HRTEM), and high-angle annular dark-field scanning TEM (HAADF STEM). It was found that the powders consist of agglomerated microparticles (Figs. 1a-1d) composed of a partially disordered $b$-AlLi phase crystallized in the NaT1 (B32) type fcc Zintle structure (space group $\mathrm{Fd} 3 \mathrm{~m}$ ), which has a homogeneity range roughly between $\mathrm{Li}$ atomic mass fractions of $45 \%$ and $56 \%$ Li [4]. STEM-EELS imaging confirmed high uniformity of the alloy (Fig. 1d). The challenge for EDXS analysis is that, in X-ray spectra of the powders, the analyzed peaks are not resolved since the $\mathrm{Li} \mathrm{Ka}(55 \mathrm{eV}) \mathrm{X}$-ray peak and $\mathrm{Al} \mathrm{L2,3}(72 \mathrm{eV}) \mathrm{X}$-ray peak are only separated by $17 \mathrm{eV}$ $[1,2]$. In the EDX spectra acquired from fresh cross-sections of the Al-Li alloy microparticles milled in the FIB, the unresolved asymmetric $\mathrm{Li} \mathrm{K} \alpha+\mathrm{Al} \mathrm{L}_{2,3}$ peak is centered at $\sim 70 \mathrm{eV}$, while the $\mathrm{Li} \mathrm{K} \alpha$ peak is centered at $59 \mathrm{eV}$ (Fig. 2a). Due to higher energy resolution of EELS as compared to EDXS, corresponding the $\mathrm{LiK}$ edge at $52 \mathrm{eV}$ and the $\mathrm{Al} \mathrm{L} 2,3$-edge at $73 \mathrm{eV}$ in the EEL spectra of the alloy powders are resolved, thus giving the atomic $\mathrm{Li} / \mathrm{Al}$ ratio of $\approx 1.3$ (Fig. $2 \mathrm{~b}$ ). The experimental detectability limits in terms of the minimum mass fraction $(\mathrm{MMF})$ of $\mathrm{Li}, \mathrm{C}_{\mathrm{MMF}}=\left(3.3 I_{\mathrm{B}}{ }^{1 / 2}\right) \times \mathrm{C}_{\mathrm{S}} / I_{\mathrm{S}}[5]$, were estimated to be $\mathrm{Li}$ atomic mass fractions of $0.11 \%$ for EDXS and $0.03 \%$ for EELS, respectively. Here $I_{\mathrm{B}}$ is the background intensity, $\mathrm{CS}_{\mathrm{S}}$ is the Li atomic fraction, and IS is the signal intensity. As expected, the proposed approach can facilitate the search for improved Li identification and quantification procedures with EDXS supported by EELS that could account for necessary experimental and theoretical parameters, chemical effects, more accurate background assignment, incident beam exposure and side reactions. Ultimately, this will help to further improve the detection limits for lithium and its compounds of practical interest. 


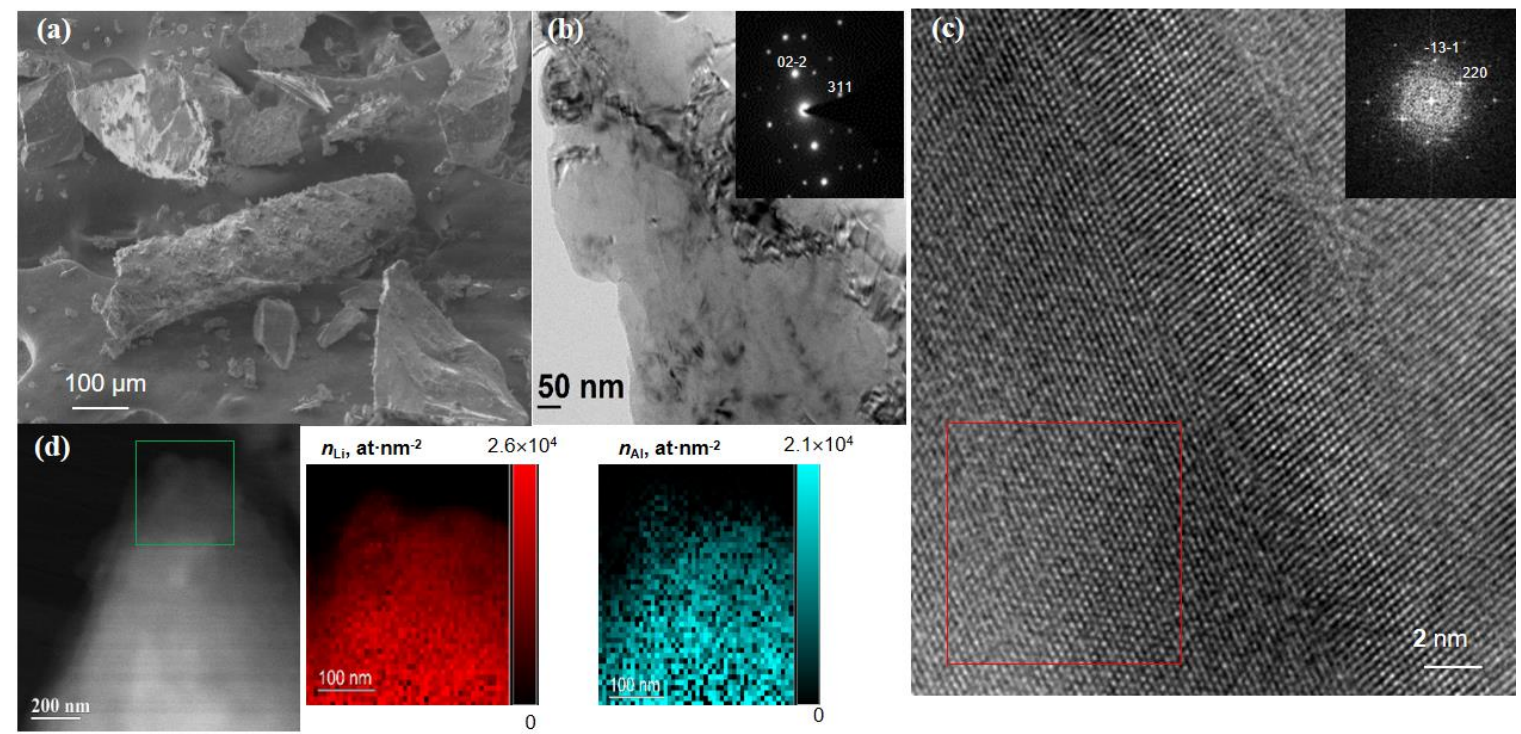

Figure 1. Al-Li alloy powder: (a) Low-voltage FESEM and (b) BF-TEM, agglomerated alloy microparticles. SAED pattern $(b$, inset) shows discrete point reflections assigned to defective b-AlLi phase. (c) HRTEM, near the edge of a twinned crystallite reveals lattice fringes from distorted b-AlLi phase. FFT from red square area (inset) indicates b-AlLi (220) and (131) point reflections, the zone axis $\mathrm{B}=$ [-114]. (d) HAADF STEM-EELS spectroscopic imaging. Color insets show areal density, $\mathrm{n}$, for lithium (red) and aluminum (cyan) in the area marked by green square.

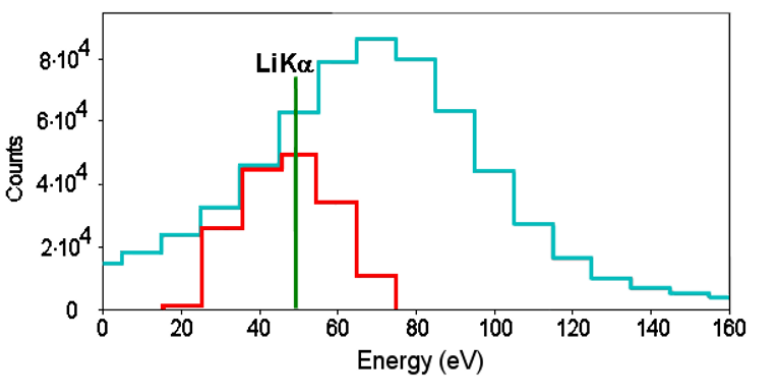

(a)

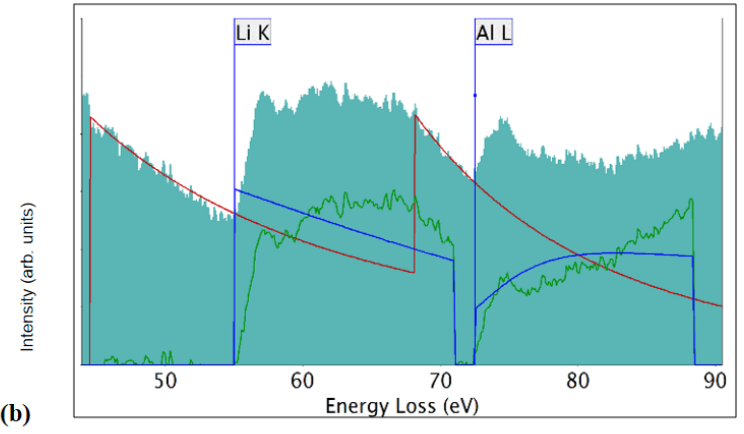

Figure 2. (a) X-ray spectrum of the Al-Li alloy powder acquired for $30 \mathrm{~s}$ at $5 \mathrm{kV}$ accelerating voltage, 200 pA probe current, the $\mathrm{Li} \mathrm{K} \alpha+\mathrm{Al} \mathrm{L2,3}$ peak at $70 \mathrm{eV}$ (cyan) overlaid with a simulated Li Ka peak at $55 \mathrm{eV}$ (red) scaled to match the experimental spectrum intensity. (b) EEL spectrum of the same powder showing the Li K-edge at $55 \mathrm{eV}$ and the $\mathrm{Al}$ L2,3 edge at $72 \mathrm{eV}$ energy loss, respectively. Fitted power law backgrounds for both edges are indicated by red line. Green curves represent the net edges separated from the extrapolated backgrounds. Blue curves show the calculated Hartree-Slater scattering cross-sections for $\mathrm{Li}$ and $\mathrm{Al}$ integrated over the same width energy windows.

\section{References}

[1] S Burgess et al, Microsc Microanal 19 (S2) (2013) 1142-1143; ibid, 22 (S3) (2016) 112-113; Microscopy Today 25 (2017) 20-28.

[2] V Oleshko et al, Microsc Microanal 24 (S1) (2018) 744-745.

[3] P Hovington et al, Scanning 38 (2016) 571-578.

[4] K Kishio, JO, Brittain, J Phys Chem Solids 40 (1979) 93W40.

[5] D Newbury, NWM Ritchie, Microsc Microanal 22 (2016) 520-535 